\title{
Data Recovery through Modulation Identification in Dense Wireless Networks
}

\author{
Anup $\mathrm{Kar}^{1}$, Aradhana Misra ${ }^{2}$, \\ Kandarpa Kumar Sarma ${ }^{3}$ and Nikos E. Mastorakis ${ }^{4}$ \\ $12{ }^{3}$ Department of Electronics and Communication Engineering, Gauhati University \\ ${ }^{4}$ Technical University of Sofia, Sofia, Kliment Ohridski 8, Bulgaria \\ 1anupkar.kar838@gmail.com, ${ }^{2}$ aradhana66@gmail.com, \\ ${ }^{3}$ kandarpaks@gauhati.ac.in, ${ }^{4}$ mastor@tu-sofia.bg
}

\begin{abstract}
With rise in device complexity and transmission rates, reliability in data recovery has become another critical issue requiring costly and computationally demanding mechanism. The popularity of artificial intelligence (AI) and its ubiquitousness have established the usefulness of design of data recovery schemes where device level complexity is less. Lower device complexity is being ensured by the use of AI driven data recovery. In this work, we focus on the design of such a mechanism where traditional process are replaced by a neuro-computing structure. The advantage is lower levels of device complexity but incorporation of a training latency. Experimental results have established the reliability of the proposed system.
\end{abstract}

Keywords : Modulation Recognition, Artificial Neural Networks, Multi Layer Perceptron

\section{INTRODUCTION}

Automatic modulation recognition (AMR)is an emerging issue in data recovery and is likely to reduce system design complexity. Of late, artificial intelligence (AI) driven AMR has received greater attention because of the fact that much of the conventional device design complexity can be considerably curtailed[1]. AMR has its roots in military communication intelligence appli- cations such as counter channel jamming, spectrum surveillance, threat evaluation, interference identifi- cation, etc [2]. Most methods proposed initially were de- signed for analogue modulations. The recent contributions in the subject focus more on digital communication especially the ones that deal with high data rates. Primarily, this is due to the increasing usage of digital modulations in many popular applications, such as mobile telephony, personal dial-up network, indoor wireless network, etc. Also, such modulation schemes provide higher data rates, bandwidth mamagement, quality control and link reliability. With the rising developments in software-defined radio (SDR) systems, automatic digital modulation recognition (ADMR) has gained more attention. Such units can act as front end to SDR systems before demodulation takes place. Thus a single SDR system can robustly handle multiple modulations and provide flexibility of operation over a range of schemes.

With rise in device complexity and transmission rates, reliability in data recovery has become another critical issue requiring costly and computationally demanding mechanism. The popularity of $\mathrm{AI}$ and its ubiquitousness have established the usefulness of design of data recovery schemes where device level complexity is less. In this background, the emergence of AI driven tools like Artificial Neural Network (ANN) comes to the forefront. It is apparent that an ANN derives its computing power through multiple ways. First, its massively parallel distributed structure and, second, its ability to learn and therefore generalize[3]. Generalization refers to the ANNs generation of reasonable outputs for inputs not encountered during training (learning). These two information processing 
capabilities make it possible for ANNs to find proper approximate solutions to complex (largescale) problems that are intractable. Furthermore, in ANNs there is no requirement of pre-processing, upsampling and downsampling.

In this work, we focus on the design of such a mechanism where traditional process are replaced by a neuro-computing structure. The advantage is lower levels of device complexity but incorporation of a training latency. Experimental results have established the reliability of the proposed system.

\section{Theoritical Considerations}

Here, we briefly discuss the relevant theoritical consideration.

\section{A. MODULATION RECOGNITION}

The recognition of the modulation format of a detected signal is the intermediate step between signal detection and demodulation [2]. With no knowledge of the transmitted data and many unknown parameters at the receiver, like the signal power, carrier frequency, phase offsets, timing information etc blind identification of the modulation is a difficult task. This becomes more challenging in real-world scenarios where multipath fading is a common occurrence with frequency selective and time varying behaviour of wireless channels [2]. Modulation recognition system must be able to make the correct classification of the modulation schemes of the received signal under interference. Automatic recognition of different modulation schemes can be done by an intelligent receiver.

\section{B. $\underline{A N N S}$}

These are computing systems inspired by the biological neural networks. Such systems "learn" (i.e. progressively improve performance on) tasks by considering examples, generally without taskspecific programming.

An ANN is based on a collection of connected units or nodes called artificial neurons (a simplified version of biological neurons in an animal brain). Each connection (a simplified version of a synapse) between artificial neurons can transmit a signal from one to another. The artificial neuron that receives the signal can process it and then signal artificial neurons connected to it.
In common ANN implementations, the signal at a connection between artificial neurons is a real number, and the output of each artificial neuron is calculated by a non-linear function of the sum of its inputs. Artificial neurons and connections typically have a weight that adjusts as learning proceeds. The weight increases or decreases the strength of the signal at a connection. Artificial neurons may have a threshold such that only if the aggregate signal crosses that threshold is the signal sent. Typically, artificial neurons are organized in layers. Different layers may perform different kinds of transformations on their inputs. Signals travel from the first (input), to the last (output) layer, possibly after traversing the layers multiple times.

The original goal of the ANN approach was to solve problems in the same way that a human brain would. However, over time, attention focused on matching specific tasks, leading to deviations from biology. ANNs have been used on a variety of tasks, including communication, computer vision, speech recognition, machine translation, social network filtering, playing board and video games and medical diagnosis.
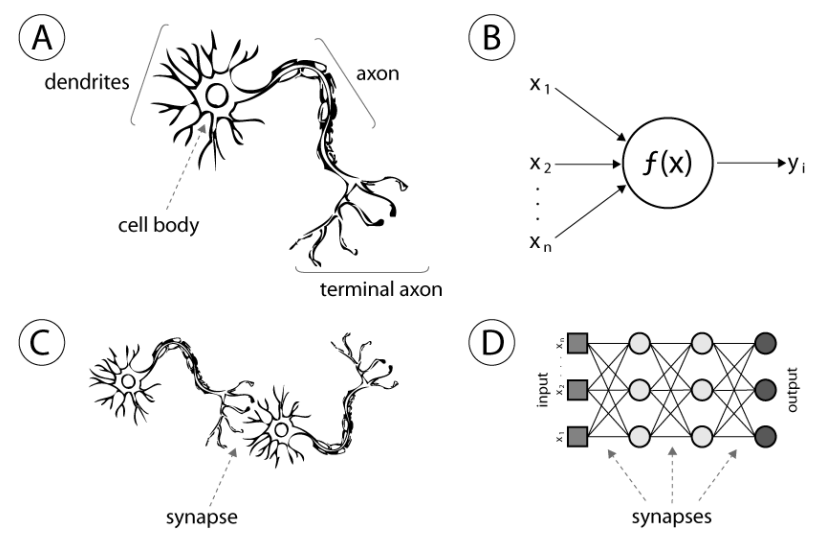

Fig. 1. The essence of Artificial Neural Network

A Multi Layer Perceptron (MLP) is a feedforward ANN model that maps a set of input data into a set of appropriate output. A MLP consists of multiple layer of nodes in a directed graph with each layer fully connected to the next one.

The number of hidden layers and nodes is somewhat arbitrary. Too few may prevent the MLP from classifying and too many may cause an unnecessarily long training time and over-fitting [3]. Overfitting occurs when the MLP is trained too long so 
that the decision boundaries get too close to the training examples. The MLP will consequently be very good for classifying the training examples, but will be very poor for classifying unseen and slightly different test examples.

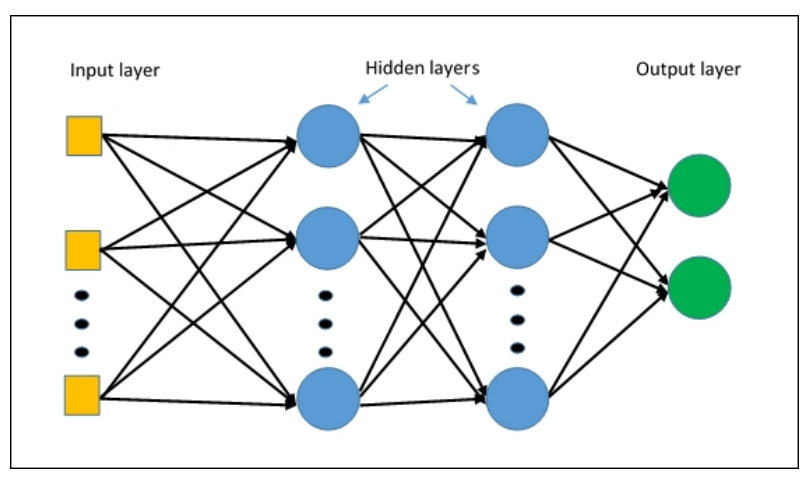

Fig. 2. Multi Layer Perceptron

\section{RELATED WORKS}

This section provides a study on previous works related to modulation recognition.

In [6] , authors focus on two feature parameters: coherence and entropy. Signal entropy and the coherence function show potential for robust recognition of HF modulation types in the presence of HF noise and multi-path.

In [7] , authors present a work related to the application of various feature parameters on real HF signals and gives guidance on which features show potential for use in robust recognition of HF modulation types in the presence of HF noise and multi-path. It also defines a measure of mean separation distance between modulation types based on an entropy parameter, and discusses the probability density function of HF noise.

In [8] , a robust Automatic modulation Classifier method for the classification of FSK, PSK, OQPSK, QAM, and amplitude-phase shift keying modulations in presence of HF noise using featurebased methods is reported.

In [9] , authors highlight a feature-based modulation recognition algorithm that is being deployed in a large-scale high-frequency (HF) communications system

In [10], the authors demonstrated that over-theair transmissions are possible: They build, train, and run a complete communications system solely composed of ANNs using unsynchronized off the shelf software-defined radios (SDRs) and opensource deep learning (DL) software libraries.

\section{Proposed Work}

A modulation recognition system is designed by following a block as shown in Figure 3. The first stage is a signal conditioning block which is required to normalize signals to certain limits to aid the learning mechanism. The classifier used for the recognition process is an ANN. The specific type selected for the work is a Multi Layer Perceptron (MLP) which is a feed forward network back propagation algorithm. The ANN is trained with signal samples of four primary modulation schemes with range of SNR variations.

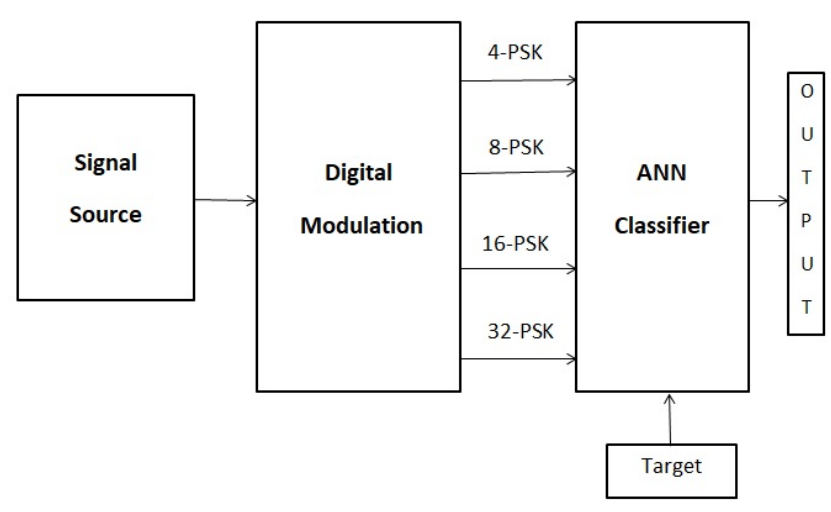

Fig. 3. Block diagram of a generic modulation recognition system

First of all a few number of data sources are selected for performing the experiment

We set selected modulation orders.

We generate required number of random symbols corresponding to modulation orders.

After modulation we pass the signal through a noisy channel.

We consider the received signal along with noise as the pattern or input. We consider pure signal that is the modulated signals as the target.

Then we train the input and target using the MLP adopting back propagation algorithm.

Then we calculate the Bit Error Rate (BER) between input and output of different modulation schemes to measure the performance.

The channel contains background variations and fluctuations due to changes in propagation conditions. For data sizes between 10 to $10^{6}$, SNR variations between 1 to $20 \mathrm{~dB}$ are considered. 
The experiments for a specific case use 4-PSK, 8-PSK, 16-PSK and 32-PSK because these are popular.

The configuration and training of the ANN is critical. The ANN with one hidden layer size of 64 with mean square error (MSE) convergence of $10^{-6}$ reaches the goal within 5 to 10 epochs. The learning is fast for which training latency is less.

\section{EXPERIMENTAL DETAILS AND DISCUSSIONS}

The work is carried out as per the process depicted in Figure 1. During training samples of M-ary PSK are taken with SNR variation between 1 to $10 \mathrm{~dB}$ and upto $60 \mathrm{~dB}$. These samples of the different modulation schemes are taken to train the ANN classifier. Training is carried out and the learning of one session is depicted as in Figure 5. The usefulness of the scheme is that the recognition is carried out despite presence of noise in the received signal content. This is due to the fact that the ANN is robust to noise and similar variations if properly trained. The specifications of the ANN are summarized in Table 1.

TABLE I

SPECIFICATIONS OF ANN USED

\begin{tabular}{|l|l|}
\hline ANN Type & MLP \\
\hline Sample Size & 4 schemes with SNR upto 30dB \\
\hline Input Size & $4 * 1000$ double \\
\hline Hidden Layer & 1 \\
\hline Activation Function & tansig,logsig \\
\hline Training Method & Back Propagation \\
\hline Epochs required(average) & 2 \\
\hline
\end{tabular}

TABLE II

Bit ERror Rate(BER)BETWEEN INPUT AND OUtPUT AT 30DB

\begin{tabular}{|l|l|}
\hline BER of 4-PSK & $1 * 10^{-4}$ \\
\hline BER of $8-P S K$ & $1 * 10^{-3}$ \\
\hline BER of 16-PSK & 0.0610 \\
\hline BER of 32-PSK & 0.0570 \\
\hline
\end{tabular}

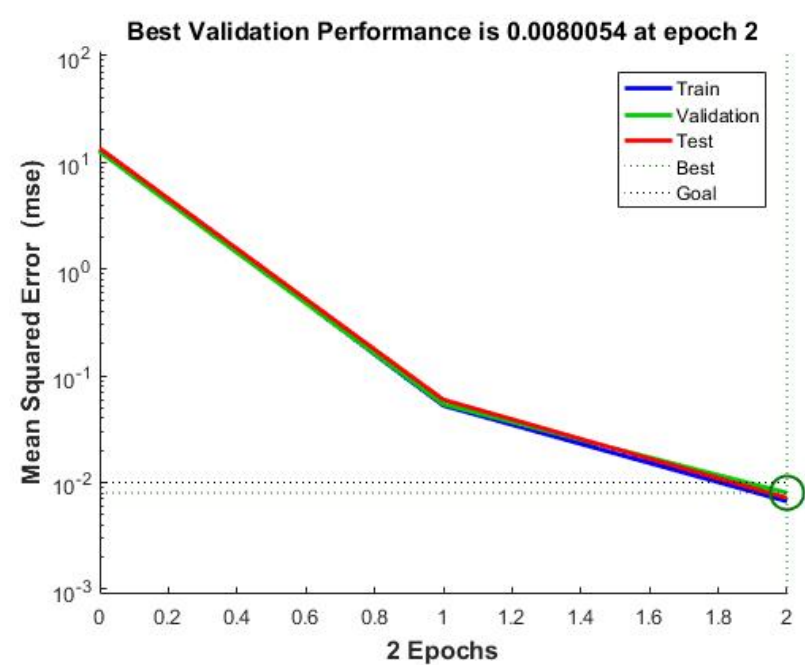

Fig. 4. ANN Convergence Plot

The error histogram is shown in Fig.6 which is generated during training. Similarly, regression plot is shown in Fig.7.

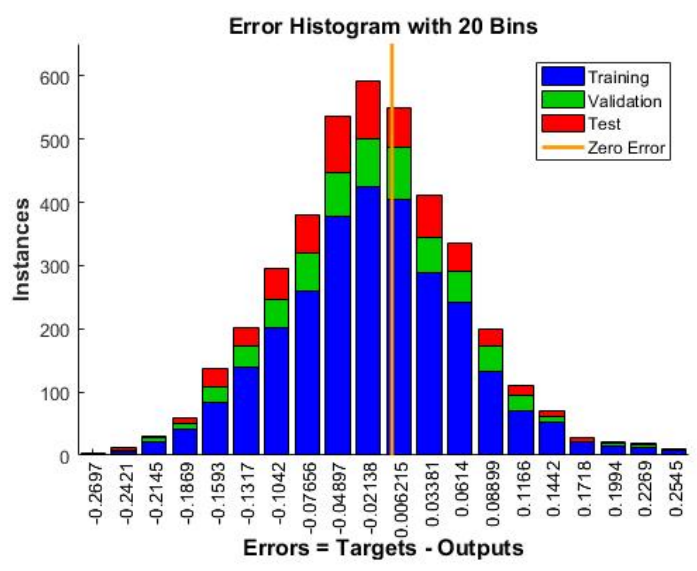

Fig. 5. Error Histogram Plot 

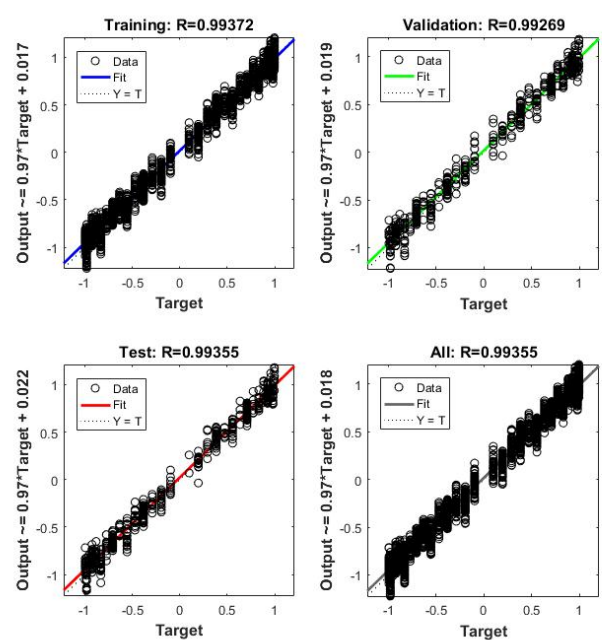

Fig. 6. Regression Plot

Scatter plots of 4 to 32-PSK recovered by the trained ANN is shown between Fig.8 to Fig.11.

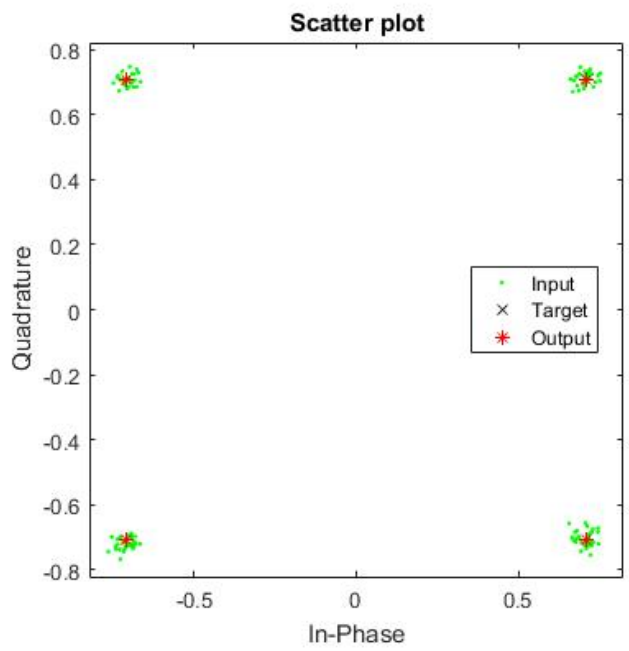

Fig. 7. Scatter Plot of 4-PSK

In an extended form, the system can be modelled to carry out recognition for a wide range of signal and modulations combinations irrespective of SNR and channel condition. It can be considered for application with system where proper knowledge of signals and modulation types are critical. Such a system can be ideal for SDR systems integrated for communication in dense wireless networks.

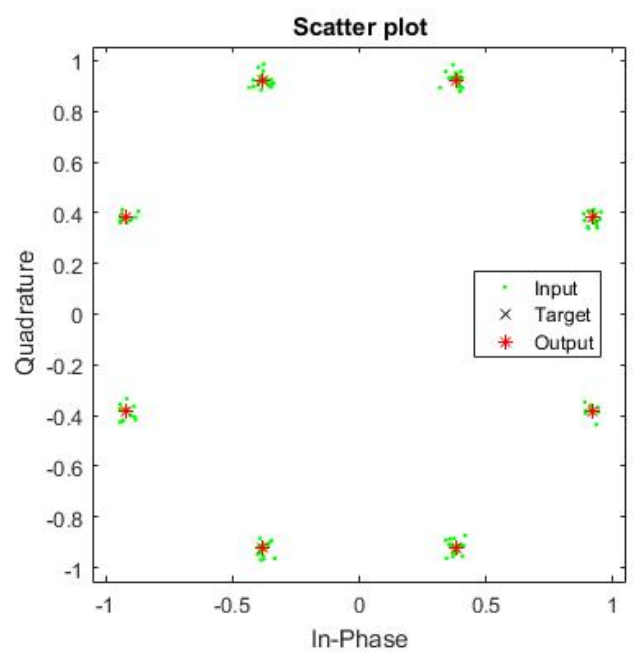

Fig. 8. Scatter Plot of 8-PSK

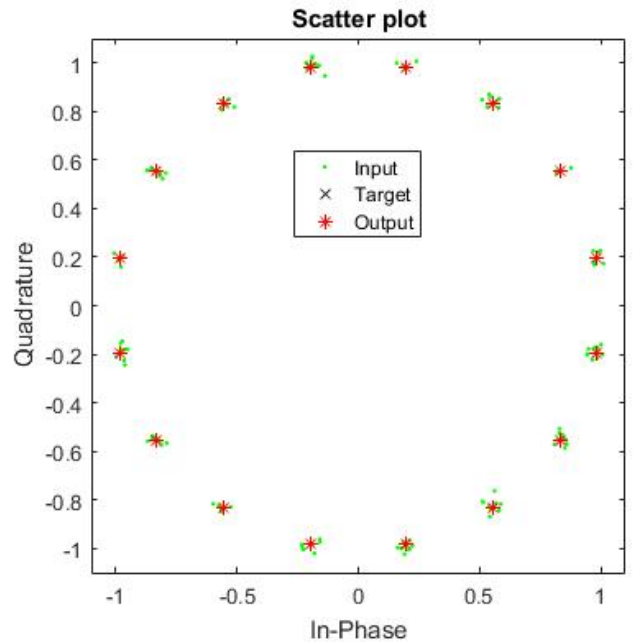

Fig. 9. Scatter Plot of 16-PSK

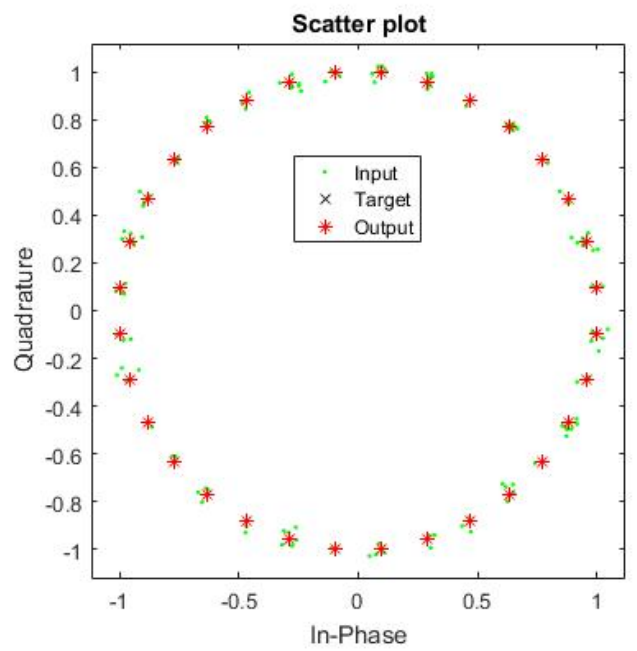

Fig. 10. Scatter Plot of 32-PSK 


\section{CONCLUSION}

Here, we have discussed the design of a data recovery scheme based on modulation identification. It is based upon a trained ANN which can discriminate signals transmitted using several modulation schemes through Gaussian and fading channels. Experimental results show that the system is reliable.

\section{REFERENCES}

[1] A. Iversen, N. K. Taylor, K. E. Brown and J. Krstad, Classification of Communication Signals and Detection of Unknown Formats Using Artificial Neural Networks, Edinburg EH 144 AS, UK:Intelligent Systems Laboratory, Heriot -Watt University, IEEE Nordic Signal Processing Symposium, pp. 6-20, 2006.

[2] M. Richterova, Signal Modulation Recognizer Based on Method of Artificial Neural Networks, China:University of Defence, Czech Republic, Progress in Electromagnetics Research Symposium, August 22-26, pp. 575-578, 2005

[3] S. Haykin, Neural Networks: A Comprehensive Foundation, 2nd ed. New Delhi, India:Pearson Education, 2003

[4] S. Haykin, Communication System , 4th ed. New Delhi, India: Wiley Publication, 2006.

[5] T. S. Rappaport, Wireless Communications: Principles and Practice, 2nd ed. New Delhi, India:Pearson Education, 2004

[6] J.E. Giesbrecht, C. Russell , and A.Derek , "Modulation recognition for HF signals." In Proc. SPIE, vol. 5649, pp. 501-512, 2004

[7] J.E. Giesbrecht, C.Russell , and A. Derek , "Modulation recognition for real HF signals." In Proc. of SPIE Vol, vol. 6035, pp. 60351S-1,2006.

[8] H. Alharbi, M. Shoaib, A. Saleh and A. Fahd , "Automatic modulation classification of digital modulations in presence of HF noise." EURASIP Journal on Advances in Signal Processing 2012, vol. 1 ,p. 238,2012

[9] J. E. Giesbrecht, "A practical modulation recognition algorithm for HF signals and beyond," 2016 IEEE 7th Annual Information Technology, Electronics and Mobile Communication Conference (IEMCON), Vancouver, BC, pp. 1-8.,2016

[10] S. Dorner,S. Cammerer, J. Hoydis, and S.T. Brink. "Deep learning-based communication over the air." arXiv preprint arXiv:1707.03384,July 11,2017

[11] J.T. O'Shea, J. Corgan, and T.C. Clancy ,'Convolutional radio modulation recognition networks." In International Conference on Engineering Applications of Neural Networks ,pp. 213-226, Springer International Publishing,September,2016

[12] S Norouzi , A Jamshidi and A.R. Zolghadrasli " Adaptive modulation recognition based on the evolutionary algorithms,"Applied Soft Computing,pp.312-319.,Jun 30,2016

[13] N.E.West and T.O Shea, "Deep architectures for modulation recognition," 2017 IEEE International Symposium on Dynamic Spectrum Access Networks (DySPAN), Piscataway, NJ, pp. 1-6.,2017

[14] T. O Shea and J. Hoydis, "An Introduction to Deep Learning for the Physical Layer," in IEEE Transactions on Cognitive Communications and Networking, vol. no. 99, pp. 1-1.,2016

[15] F. Liang, C. Shen and F.Wu, "An Iterative BP-CNN Architecture for Channel Decoding", arXiv preprint arXiv:1707.05697,18 July,2017
[16] X. Glorot, A. Bordes, and Y. Bengio. "Deep sparse rectifier neural networks." In Proceedings of the Fourteenth International Conference on Artificial Intelligence and Statistics,pp. 315-323,Jun14,2011

[17] J.T. O Shea and C. Johnathan, "Convolutional radio modulation recognition networks." In International Conference on Engineering Applications of Neural Networks,Springer International Publishing,pp. 213-226., 2016

[18] N.Samuel, T. Diskin, and A. Wiesel. "Deep MIMO Detection." arXiv preprint arXiv:1706.01151,Jun 4,2017.

[19] J.T.O'Shea, K. Kiran and T. C. Clancy, "Learning to communicate: Channel auto-encoders, domain specific regularizers, and attention." In Signal Processing and Information Technology (ISSPIT), 2016 IEEE International Symposium , pp. 223-228., 2016.

[20] J.T.O'Shea, E.Tugba , and T.C. Clancy. "Deep learning based mimo communications." arXiv preprint arXiv:1707.07980, 2017.

[21] N.Farsad and A.Goldsmith. "Detection Algorithms for Communication Systems Using Deep Learning." arXiv preprint arXiv:1705.08044, 2017.

[22] Y.LeCu, Y. Bengio, and G. Hinton. "Deep learning." Nature 521, vol. 7553 , pp.436-444,2015

[23] J.Schmidhuber "Deep learning in neural networks: An overview." Neural networks vol.61,pp. 85-117,2015.

[24] Y.Bengio "Learning deep architectures for AI." Foundations and trends in Machine Learning 2, vol. 1 ,pp. 1-127,2009.

[25] A.Ali,Y. Fan, and L.Shu, "Automatic modulation classification of digital modulation signals with stacked autoencoders." Digital Signal Processing,vol. 71,pp. 108-116,2017. 